\title{
EVALUATION OF CARTRIDGE BASED NUCLEIC ACID AMPLIFICATION TEST IN DIAGNOSIS OF PULMONARY TUBERCULOSIS
}

\author{
Subhasis Mukherjee", Debabani Biswas², Shabana Begum³, Priyanka Ghosh ${ }^{4}$, Amitava Paul5, Supriya Sarkar 6 \\ ${ }_{1}^{1}$ Assistant Professor, Department of Respiratory Medicine, College of Medicine and Sagore Dutta Hospital. \\ ${ }^{2}$ Assistant Professor, Department of Respiratory Medicine, College of Medicine and Sagore Dutta Hospital. \\ ${ }^{3}$ Demonstrator, Department of Anatomy, Medical College, Kolkata. \\ ${ }^{4}$ RMO-Cum-Clinical Tutor, Department of Respiratory Medicine, College of Medicine and Sagore Dutta Hospital. \\ ${ }_{5}^{5}$ RMO-Cum-Clinical Tutor, Department of Respiratory Medicine, College of Medicine and Sagore Dutta Hospital. \\ ${ }^{6}$ Professor, Department of Respiratory Medicine, College of Medicine and Sagore Dutta Hospital.
}

\begin{abstract}
BACKGROUND

Tuberculosis still continues to be one of the commonest cause of infectious disease related morbidity in the developing world. Diagnosis of pulmonary tuberculosis is mostly relied on sputum smear microscopy and sputum for mycobacterial culture. In recent years, cartridge based nucleic acid amplification test (CBNAAT) has been recommended by World Health Organization as a rapid diagnostic test for detection of tuberculosis and rifampicin resistance.

The aim of this study is to determine the diagnostic yield of CBNAAT in pulmonary tuberculosis (PTB) and to compare its efficacy between sputum smear-positive and sputum smear-negative pulmonary tuberculosis.
\end{abstract}

\section{MATERIALS AND METHODS}

A prospective observational study was conducted in the Department of Respiratory Medicine in a teaching hospital in eastern India for a period of one year. All re-treatment cases of PTB, newly detected smear-negative PTB and all human immunodeficiency virusPTB co-infected cases were recruited for the study and sputum for CBNAAT was performed in all of them. Results were analysed in light of diagnostic yield of CBNAAT with special emphasis on comparing CBNAAT results between different subgroups- sputum smear-positive, sputum smear-negative PTB and immunocompromised patients.

\section{RESULTS}

Out of 228 cases of PTB, 190 were sputum smear negative and 38 cases were sputum smear positive. Mean age of the study population was $33 \pm 17.13$ years. Sensitivity of sputum smear for AFB was $16.67 \%$ (CI-12.07\%-22.15\%) with a negative predictive value (NPV) of $20.83 \%$ (CI- 19.89\%-21.81\%). Sputum smear negativity was found to be more common in females. CBNAAT was positive in 111 cases. Overall, sensitivity of CBNAAT was 48.68\% (95\% CI- 42.03\%-55.37\%). Sensitivity of CBNAAT varied significantly between sputum smear-positive PTB (100\%, CI- 92.89\%-100\%) and sputum smear-negative PTB (38.42\%; CI31.47\%-45.74\%) (p- <0.0001). Sensitivity of CBNAAT was 33.33\% (CI-17.96\%-51.83\%) in PTB-HIV co-infected patients. Rifampicin resistance was detected in five $(2.2 \%)$ patients with $100 \%$ sensitivity and specificity.

\section{CONCLUSION}

CBNAAT adds significantly to the diagnostic yield of PTB in comparison to sputum smear microscopy, but its sensitivity is lower in sputum smear-negative cases. It has additional advantage of identifying rifampicin resistance with high sensitivity and specificity.

\section{KEYWORDS}

Pulmonary Tuberculosis, Nucleic Acid Amplification Test, Smear Positive, Smear Negative, Human Immunodeficiency Virus.

HOW TO CITE THIS ARTICLE: Mukherjee S, Biswas D, Begum S, et al. Evaluation of cartridge based nucleic acid amplification test in diagnosis of pulmonary tuberculosis. J. Evolution Med. Dent. Sci. 2017;6(74):5281-5286, DOI: 10.14260/Jemds/2017/1147

\section{BACKGROUND}

Pulmonary tuberculosis is still one of the commonest cause of infectious disease related morbidity and mortality in the developing countries.[1] Diagnosis of pulmonary tuberculosis (PTB) mostly relies on identification of acid-fast bacilli (AFB) in sputum smear, but its limitation is low sensitivity.[2,3] Conventional mycobacterial cultures (Solid culture in Lowenstein-Jensen medium) takes about 6-8 weeks' time;

Financial or Other, Competing Interest: None.

Submission 24-08-2017, Peer Review 05-09-2017,

Acceptance 08-09-2017, Published 14-09-2017.

Corresponding Author:

Dr. Debabani Biswas,

\#607, Purbalok, Kalikapur,

Plot No. 328, Kolkata-700099, West Bengal.

E-mail: debabanibiswas@gmail.com

DOI: $10.14260 /$ jemds $/ 2017 / 1147$ newer liquid culture methods like BACTEC or Mycobacterial growth indicator tube (MGIT) gives relatively rapid results but is costly. ${ }^{[4,5]}$ Cartidge based nucleic acid amplification test (CBNAAT) is a nested polymerase chain reaction (PCR) technique that identifies small quantities of genetic elements of Mycobacterium tuberculosis from clinical specimens and it can identify resistance to rifampicin, the surrogate marker of multi-drug resistant (MDR) tuberculosis, at the same time. CBNAAT is completely automated, has minimal biosafety hazard and can give result within two hours. World Health Organization has endorsed the use of this rapid molecular diagnostic test for diagnosis of tuberculosis with special emphasis on drug-resistant tuberculosis, human immunodeficiency virus (HIV) and TB co-infection, paediatric tuberculosis, extrapulmonary tuberculosis and smearnegative pulmonary tuberculosis.[6,7] There has been paucity of data from eastern India regarding diagnostic role of CBNAAT specially in diagnosis of sputum smear-negative PTB 
cases. In this background, the present study was carried out to determine the diagnostic role of CBNAAT in PTB except in new microbiologically confirmed cases without HIV coinfection.

\section{MATERIALS AND METHODS}

A prospective, observational study of all adult cases (above 12 years of age), of new sputum smear negative, clinically diagnosed PT, all TB-HIV co-infected patients and all retreatment cases of pulmonary tuberculosis, admitted or attending outpatients department in the Department of Respiratory Medicine of a teaching Hospital in Kolkata was carried out over a period of one year (July 2016 - June 2017).

\section{Clinically Diagnosed TB Case [8]}

Presumptive Pulmonary TB: refers to a person with any of the symptoms and signs suggestive of PTB including cough $>2$ weeks, fever $>2$ weeks, significant weight loss, haemoptysis, abnormality in chest radiograph.

Microbiologically confirmed Pulmonary tuberculosis: refers to a presumptive pulmonary TB patient with sputum positive for acid-fast bacilli or positive for Mycobacterium tuberculosis on culture, or positive for tuberculosis through quality assured rapid molecular diagnostic test.

\section{Clinically Diagnosed TB Case}

Refers to a presumptive pulmonary TB patient who is not microbiologically confirmed, but has been diagnosed with active TB by a clinician on the basis of clinical findings and having radiological lesions consistent with active parenchymal tuberculosis on chest x-ray/CT scan of the thorax (nodular consolidation with or without cavity in apex, tree in bud appearance).

Written informed consent was taken from all patients and the study was cleared by the institute's ethics committee.

\section{Inclusion Criteria}

1. All cases of clinically diagnosed sputum smear-negative pulmonary tuberculosis.

2. All re-treatment cases of microbiologically confirmed pulmonary tuberculosis (recurrent, failure, treatment after loss to follow-up).

3. All presumptive pulmonary tuberculosis cases with HIV co-infection.

\section{Exclusion Criteria}

1. Age less than 12 years;

2. New microbiologically confirmed PTB patients without HIV co-infection;

3. Patients not giving consent for the study.

\section{Study Protocol}

All patients who fulfilled the case definition and inclusion criteria and who consented for the study were considered for subsequent investigation and analysis. Patients were evaluated for clinico-demographical parameters such as age, sex, symptoms with duration, comorbidities, sputum smear status for acid-fast bacilli (AFB). Two samples of sputum were collected from each patient in Falcon tubes designated for sample collection for CBNAAT and were sent for CBNAAT at B.N. Bose Hospital, Kolkata as per Revised National Tuberculosis Control Programme (RNTCP) protocol for testing for CBNAAT for Mycobacterium tuberculosis (Cepheid, GX-IV Processing Unit: 11.00" w x 12.00" h x 11.70" d, GXIV-4-D) [6, 8, 9]. Sputum samples for CBNAAT was also sent in 40 cases of community-acquired pneumonia and 10 cases of suspected lung nodule/mass lesion to look for false positive results but CBNAAT result was negative in all 50 cases. Chest X-ray posteroanterior (PA) view was done in all patients of microbiologically confirmed and clinically diagnosed PTB cases. High-resolution Computed Tomography (HRCT) scan of thorax with contrast was done additionally in clinically diagnosed PTB cases for obtaining some greater anatomical details regarding radiological evidence of active pulmonary tuberculosis (Nodular consolidation with or without cavity in apex, tree in bud appearance, bilateral involvement). Blood were sent for testing for HIV infection at the integrated counselling and testing centre (ICTC) of our hospital. Relevant haematological investigations like fasting blood sugar, complete haemogram, urea, creatinine and baseline liver function test were also done in all patients.

sA composite diagnostic index (Comprising of sputum AFB smear and/or radiology, and/or clinical judgement, and/or response to antitubercular drugs) was considered as the reference standard for diagnosis of pulmonary tuberculosis in this study and result of CBNAAT was compared with that composite diagnostic index.

\section{Statistical Analysis}

Statistical analyses were performed using SPSS version 20.0 (SPSS Inc., Chicago, IL) software for MS-Windows. Descriptive frequencies were expressed using mean and standard deviation. Sensitivity, specificity, positive predictive value (PPV), negative predictive value (NPV) and negative likelihood ratio were calculated with $95 \%$ confidence interval (CI) where relevant. P value was calculated using Fisher's exact test and Chi Square test as applicable and $P$ value of less than 0.05 was considered significant.

\section{RESULTS \\ Categorisation of Pulmonary TB Cases}

A total of 228 cases of microbiologically confirmed or clinically diagnosed PTB were encountered during the study period, of which 179 cases were new cases of clinically diagnosed PTB with negative sputum smear and 49 were retreatment PTB cases. Twenty of the re-treatment cases were recurrent PTB, 11 were treatment after loss to follow- up, seven were treatment after failure and 11 belonged to other previously treated category. So, overall 190 cases had sputum smear for AFB negative and 38 cases were tested positive for AFB in sputum.

Among 190 cases of sputum smear negative clinically diagnosed PTB cases, 33 were PTB with HIV co-infection, 11 were other previously treated cases and rest 146 were immunocompetent new sputum smear-negative PTB.

\section{Demographic Profile}

Overall mean age of PTB patients in the study population was 33 years \pm 17.13 years (mean \pm SD). $59.2 \%$ (135 out of 228 ) patients were male. Mean age in the female population was slightly lower (28.23 \pm 15.63 years) compared to the mean age in the male group (36.35 \pm 17.3 years). There was no significant difference in mean age between sputum smearpositive PTB and sputum smear-negative PTB population, but 
sputum smear-negative PTB was significantly more prevalent in females in the study population (p-0.04) [Table 1]. Out of 190 cases of sputum smear-negative PTB cases, 82 (43.16\%) were females, in contrast to $11(28.95 \%)$ females out of 38 sputum smear-positive PTB. Diabetes mellitus was the commonest comorbidity, being found in 15 (7.89\%) cases.

\section{Microbiologic Diagnostic Spectrum}

Sputum smear for AFB was positive in $16.67 \%$ (38 out of 228 cases). Sputum for CBNAAT detected Mycobacterium tuberculosis in 111 patients. CBNAAT was found to be positive in all $38(100 \%)$ cases of sputum smear-positive PTB, but in 73 out of 190 (38.42\%) cases of sputum smearnegative PTB $(\mathrm{p}-<0.0001)$ [Table 2]. Thus, sputum for CBNAAT testing resulted in a relative increase in detection rate of microbiologically confirmed tuberculosis cases by $32.02 \%$ compared to sputum smear microscopy.

Overall, in this study population, sensitivity of sputum smear for AFB was only $16.67 \%$ (95\% CI- 12.07\%-22.15\%) with specificity of $100 \%$, negative predictive value (NPV) of $20.83 \%$ (95\% CI- 19.89\%-21.81\%) and negative likelihood ratio of 0.83 (95\% CI- 0.79-0.88). On the other hand, against a composite diagnostic index of PTB (comprising of sputum AFB smear, and/or chest radiograph and/or HRCT Thorax, and/or clinical judgement, and/or response to antitubercular drugs), overall sensitivity of sputum CBNAAT was $48.68 \%$ (95\% CI- $42.03 \%-55.37 \%$ ), with specificity of $100 \%$ (95\% CI-
92.89\%-100\%). Sensitivity of CBNAAT was found to be $100 \%$ ( $95 \%$ CI $-90.75 \%-100 \%$ ) for microbiologically confirmed PTB, but sensitivity came down to $38.42 \%$ (95\% CI- 31.47\%$45.74 \%)$ for sputum smear-negative PTB $(\mathrm{p}-<0.0001)$ [Table 3]. Specificity were $100 \%$ (95\% CI- $92.89 \%-100 \%$ ) in both the groups. Sputum for CBNAAT was found positive in 93.88\% (46 out of 49) of re-treatment cases of PTB, on the contrary, it was positive in $36.31 \%$ (65 of 179) of newly diagnosed sputum smear-negative PTB ( $\mathrm{p}-<0.0001)$.

On detailed analysis of sputum smear-negative PTB group, CBNAAT showed detection of Mycobacterium tuberculosis in $36.99 \%$ (54 of 146) of immunocompetent newly smear negative cases, $33.33 \%$ (11 of 33) of HIV infected smear negative patients and $72.72 \%$ ( 8 of 11) of other previously treated cases [Table 4]. There was no significant difference in yield of sputum CBNAAT between immunocompetent and immunocompromised patients ( $p$ 0.56 ) but sputum microscopy yield was significantly lower in immunocompromised group (p-0.01).

Sputum for CBNAAT detected "Rifampicin resistance" in five patients-two of them were in the re-treatment group (treatment after lost to follow-up), two were new case of PTB with HIV co-infection and one was new sputum smearnegative PTB without HIV infection. All these cases of rifampicin resistance were also confirmed to be MDR cases by Line probe assay (LPA).

\begin{tabular}{|c|c|c|}
\hline & Sputum smear-positive PTB (n=38) & Sputum smear-negative PTB (n=190) \\
\hline Age (mean \pm SD) in years & $32.97 \pm 13.64$ & $33.005 \pm 17.71$ \\
\hline Male: Female ratio & $2.45: 1$ & $1.30: 1$ \\
\hline Diabetes mellitus & 7 & 8 \\
\hline HIV infection & 0 & 33 \\
\hline \multicolumn{2}{|c|}{ Table 1. Demographic Characters } \\
\hline
\end{tabular}

\begin{tabular}{|c|c|c|}
\hline & Sputum AFB Smear positive $(\mathrm{n}=38)$ & Sputum AFB Smear Negative (n=190) \\
\hline Sputum CBNAAT positive (n=111) & 38 & 73 \\
\hline Sputum CBNAAT negative (n=117) & 0 & 117 \\
\hline \multicolumn{2}{|c|}{ Table 2. Diagnostic Yield of CBNAAT versus Sputum Smear Microscopy } \\
\hline
\end{tabular}

\begin{tabular}{|c|c|c|c|c|c|}
\hline & $\begin{array}{c}\text { Sensitivity } \\
(\mathbf{9 5 \%} \text { CI) }\end{array}$ & $\begin{array}{c}\text { Specificity } \\
\mathbf{( 9 5 \%} \mathbf{C I})\end{array}$ & $\begin{array}{c}\text { Positive predictive } \\
\text { value (PPV) }\end{array}$ & $\begin{array}{c}\text { Negative } \\
\text { Predictive value } \\
\text { (NPV) }\end{array}$ & $\begin{array}{c}\text { Negative Likelihood } \\
\text { Ratio }\end{array}$ \\
\hline Overall PTB & $\begin{array}{c}48.68 \% \\
(42.03 \%-55.37 \%)\end{array}$ & $\begin{array}{c}100 \% \\
(92.89 \%-100 \%)\end{array}$ & $100 \%$ & $29.94 \%$ & 0.51 \\
$(27.36 \%-32.66 \%)$ & $100 \%$ & \\
\hline $\begin{array}{c}\text { Sputum smear- } \\
\text { positive PTB }\end{array}$ & $100 \%$ & $\begin{array}{c}100 \% \\
(92.89 \%-100 \%)\end{array}$ & $100 \%$ & $29.94 \%$ & $0.58)$ \\
\hline $\begin{array}{c}\text { Sputum smear- } \\
\text { negative PTB }\end{array}$ & $\begin{array}{c}38.42 \% \\
(31.47 \%-45.74 \%)\end{array}$ & $\begin{array}{c}100 \% \\
(92.89 \%-100 \%)\end{array}$ & $100 \%$ & $0.55-0.69)$ \\
\hline \multicolumn{7}{|c|}{ Table 3. Diagnostic Sensitivity and Specificity of Sputum CBNAAT in PTB } \\
\hline
\end{tabular}

\begin{tabular}{|c|c|c|c|c|c|}
\hline & $\begin{array}{c}\text { Sensitivity } \\
(\mathbf{9 5 \%} \text { CI) }\end{array}$ & $\begin{array}{c}\text { Specificity } \\
(\mathbf{9 5 \%} \text { CI) }\end{array}$ & PPV & NPV & $\begin{array}{c}\text { Negative } \\
\text { Likelihood Ratio }\end{array}$ \\
\hline $\begin{array}{c}\text { Smear negative PTB } \\
\text { immunocompetent } \\
(\mathrm{n}=157)\end{array}$ & $\begin{array}{c}39.49 \% \\
(31.79 \%-47.59 \%)\end{array}$ & $\begin{array}{c}100 \% \\
(92.89 \%-100 \%\end{array}$ & $100 \%$ & $\begin{array}{c}34.48 \% \\
(31.69 \%-37.39 \%)\end{array}$ & $\begin{array}{c}0.61 \\
(0.53-0.69)\end{array}$ \\
\hline $\begin{array}{c}\text { Smear negative PTB } \\
\text { immunocompromised }\end{array}$ & $\begin{array}{c}33.33 \% \\
(17.96 \%-51.83 \%)\end{array}$ & $\begin{array}{c}100 \% \\
(92.89 \%-100 \%\end{array}$ & $100 \%$ & $\begin{array}{c}69.44 \% \\
(64.10 \%-74.31 \%)\end{array}$ & $\begin{array}{c}0.67 \\
(0.52-0.85)\end{array}$ \\
\hline \multicolumn{6}{|c|}{ Table 4. Diagnostic Yield of CBNAAT in Smear-negative PTB- Immunocompetent Versus Immunocompromised Patients } \\
\hline
\end{tabular}




\begin{tabular}{|c|c|c|c|c|c|c|}
\hline \multirow{2}{*}{ Name of the Study } & \multicolumn{2}{|c|}{ Overall PTB } & Smear-Positive PTB & \multicolumn{2}{c|}{ Smear-negative PTB } \\
\cline { 2 - 7 } & Sensitivity & Specificity & Sensitivity & Specificity & Sensitivity & Specificity \\
\hline Dewan R et al & $40.00 \%$ & $100 \%$ & $100 \%$ & $100 \%$ & $32.58 \%$ & $100 \%$ \\
\hline Theron G et al & $78.7 \%$ & $94.4 \%$ & $94.7 \%$ & $95 \%$ & $46.8 \%$ & $94.4 \%$ \\
\hline Geleta et al & $65.5 \%$ & $96.3 \%$ & $95.2 \%$ & $96.3 \%$ & $48.6 \%$ & $96.3 \%$ \\
\hline Agarwal M et al & $86.8 \%$ & $93.1 \%$ & $100 \%$ & $90 \%$ & $79.1 \%$ & $93.1 \%$ \\
\hline Sharma SK et al & $95.7 \%$ & $99.6 \%$ & $99.2 \%$ & $99.6 \%$ & $77.7 \%$ & $99.6 \%$ \\
\hline Sowjanya DS et al & $70.24 \%$ & $100 \%$ & $99.08 \%$ & $100 \%$ & $37.50 \%$ & $100 \%$ \\
\hline Boehme CC et al & $92.2 \%$ & $99.2 \%$ & $98.2 \%$ & $99.2 \%$ & $72.5 \%$ & $99.2 \%$ \\
\hline \multicolumn{3}{|c|}{ Table 5. Sensitivity and Specificity of CBNAAT-Results of Different Studies } \\
\hline
\end{tabular}

\section{DISCUSSION}

India accounts for around one-fourth of the global tuberculosis cases.[10] Detection of AFB in sputum smear is a simple, rapid, inexpensive and very specific for diagnosis for PTB, its limitation is its low sensitivity.[2,3] Sputum culture for Mycobacterium tuberculosis is more sensitive and specific, but it takes 2-8 weeks' time depending on the method used and is costly.[4,5] Chest $\mathrm{x}$-ray is neither sensitive nor specific for diagnosis of PTB.[11] So, there was a long felt need for a newer rapid diagnostic test for PTB with improved sensitivity and specificity. WHO has endorsed the use of CBNAAT as a rapid diagnostic test for diagnosis of tuberculosis and prioritised areas like drug-resistant tuberculosis, paediatric tuberculosis, TB-HIV co-infection, extrapulmonary tuberculosis and sputum smear-negative PTB for use of CBNAAT.[6]

In this study, mean age of PTB patients was 33 years \pm 17.13 years (mean \pm SD) with slight male preponderance $(59.2 \%)$. Dewan et al[12] have also reported that mean age of patients in their study was $35 \pm 9$ years, $69 \%$ of their patients were in $20-40$ years age group and $76 \%$ were male. Sputum smear-negative PTB was found to be more common in females. Diabetes mellitus ( $\mathrm{n}=15$ ) was a common comorbidity in our study population. Increased prevalence of diabetes mellitus and tuberculosis is well documented in the published literature.[13,14] HIV infection was seen in 33 cases $(14.47 \%)$ with male predominance $(57.6 \%)$. Dewan et al[12] and Theron $\mathrm{G}$ et al[15] have reported a higher value of TB-HIV co-infection of $40 \%$ and $27.08 \%$ respectively in their studies, though both the studies were conducted in high HIV prevalence setting.

Sensitivity of sputum smear for AFB was very low (16.67\%) with a low NPV (20.83\%) in excluding PTB in this study. Geleta DA et al[16] have also found a very low sensitivity $(9.3 \%)$ of conventional sputum smear microscopy by Ziehl-Neelsen staining. CBNAAT showed an advantage of increase in diagnosis of microbiologically confirmed PTB cases by $32 \%$ over and above the cases diagnosed by sputum smear for AFB. Dewan R et al[12] and Geleta DA et al[16] have also mentioned an increase in microbiologically confirmed PTB diagnosis by $29 \%$ and $31 \%$ respectively by CBNAAT compared to sputum smear microscopy.

Overall, CBNAAT was positive in $48.68 \%$ (95\% CI42.03\%-55.37\%) PTB cases in this study, but its result varied significantly between $100 \%$ (95\% CI $-90.75 \%-100 \%)$ in sputum smear-positive PTB and 38.42\% (95\% CI- 31.47\%$45.74 \%$ ) in sputum smear-negative PTB. Similar results of very high sensitivity of CBNAAT in smear positive cases have been reported by most of the studies, but sensitivity of CBNAAT in smear negative cases varied between 35\%-79.1\% among several studies [Table 5].[12,15,16-20] Specificity of CBNAAT was $100 \%$ (95\% CI- 92.89\%-100\%) in this study in both smear positive and smear negative group and is in line with other studies which have also demonstrated a very high specificity of CBNAAT in diagnosis of pulmonary tuberculosis [Table 5].[12,15,16-20]

Sensitivity of sputum smear microscopy was even lower in this study in patients infected with HIV, all of the TB-HIV co-infected patients were sputum smear negative in comparison to 38 out of 195 immunocompetent patients being sputum smear-positive on microscopy (p-0.01). However, there was no statistically significant difference in sensitivity of CBNAAT between sputum smear-negative PTB cases with or without HIV co-infection ( $p-0.56)$. These findings were similar to the observations of Van Rle A et al ${ }^{[21]}$ and Carriquiry $\mathrm{G}^{[22]}$ et al in their respective studies. In this study, sputum for CBNAAT resulted in an increase in microbiologically confirmed TB cases by $33.3 \%$ in HIV infected patients. Dewan R et al[12] have also reported a $29 \%$ increase in diagnosis of PTB by application of sputum CBNAAT over sputum smear microscopy in HIV infected patients. Among all smear negative cases, sensitivity of CBNAAT was found to be significantly higher $(72.73 \%$; $95 \%$ CI -39.03\%-93.98\%; p value- 0.023 ) with NPV of $94.34 \%$ and negative likelihood ratio of 0.27 in cases of sputum smearnegative tuberculosis with previous history of antitubercular drug intake.

Rifampicin resistance was detected in five (2.2\%) cases of PTB. Two of these five cases were sputum smear-positive with previous history of antitubercular treatment default; two were sputum smear-negative PTB with HIV co-infection and one patient was a new case of sputum smear-negative PTB. Sensitivity (100\%; 95\% CI-47.82\%-100\%), specificity (100\%; 95\% CI- 98.36\%-100\%), PPV (100\%) and NPV $(100 \%)$ of CBNAAT for identifying rifampicin resistance were very high in this study. All of the five cases of rifampicinresistant PTB in this study were confirmed as MDR-TB cases by LPA, so sensitivity and specificity of CBNAAT as a surrogate marker of MDR TB was also 100\%. This finding has been supported by the study of Sharma SK et al,[18] where sensitivity and specificity of CBNAAT was found to be $94.5 \%$ 99\% and $97.7 \%-99.3 \%$ respectively. Theron $\mathrm{G}$ et al[15] have also shown that with the use of second generation software for identifying rifampicin resistance, specificity and NPV for rifampicin resistance were $100 \%$ by CBNAAT. Rifampicin resistance on CBNAAT has been found to be a good surrogate marker for MDR-TB in their study, too, as $90 \%$ of rifampicinresistant cases were confirmed as MDR by subsequent analysis. Although, sputum for CBNAAT is very good in rapidly identifying Rifampicin resistance in PTB patients, a study from Mumbai have reported there are good number of Isoniazid resistant but Rifampicin sensitive cases, where only relying on CBNAAT result may lead to missing out of drugresistant PTB cases.[23] 
Limitation of this study was that we did not compare CBNAAT with sputum for mycobacterial culture, the current gold standard for diagnosis of PTB. Future studies in this field comparing result of CBNAAT with mycobacterial cultures are needed.

\section{CONCLUSION}

CBNAAT is a very useful and rapid test for diagnosis of PTB, but its limitation is that its sensitivity is modest in smearnegative PTB with or without HIV co-infection in comparison to very high sensitivity in smear-positive PTB. In spite of its modest sensitivity in smear-negative PTB and TB-HIV coinfection, in terms of absolute numbers, CBNAAT adds significantly to the number of microbiologically confirmed PTB in these patients. Main advantage of CBNAAT lies in its ability in rapid diagnosis and early detection of rifampicin resistance. Sputum for CBNAAT should be sent in all cases of TB-HIV co-infection, sputum smear-negative PTB and all retreatment cases of PTB for an early and confident diagnosis of PTB and to look for rifampicin resistance.

\section{ACKNOWLEDGEMENTS}

1. Dr. Sonali Das, District Tuberculosis Officer, North 24 Parganas. 2. Mr. Chandan Mondal, Senior Treatment and Laboratory, Supervisor, RNTCP, College of Medicine and Sagore Dutta Hospital. 3. Mr. Rajib Chatterjee, Laboratory Technician, RNTCP, B.N. Bose Hospital, Barrackpore.

\section{REFERENCES}

[1] World Health Organization. Global tuberculosis report Geneva: WHO, 2014. http://apps.who.int/iris/ bitstream/10665/137094/1/9789 241564809_eng. pdf?ua $=1$.

[2] Hopewell PC, Pai M, Maher D, et al. International standards for tuberculosis care. Lancet Infect Dis 2006;6(11):710-25.

[3] Getahun H, Harrington M, O'Brien R, et al. Diagnosis of smear negative pulmonary tuberculosis in people with HIV infection or AIDS in resource-constrained settings: informing urgent policy changes. Lancet 2007;369(9578):2042-9.

[4] Moore DF, Guzman JA, Mikhail LT. Reduction in turnaround time for laboratory diagnosis of pulmonary tuberculosis by routine use of a nucleic acid amplification test. Diagn Microbiol Infect Dis 2005;52(3):247-54.

[5] Pai M, Kalantri S, Dheda K. New tools and emerging technologies for the diagnosis of tuberculosis: part II. Active tuberculosis and drug resistance. Expert Rev Mol Diagn 2006;6(3):423-32.

[6] Automated real-time nucleic acid amplification technology for rapid and simultaneous detection of tuberculosis and rifampicin resistance: Xpert MTB/RIF system for the diagnosis of pulmonary and extrapulmonary TB in adults and children: policy update. Geneva: World Health Organization, 2013. http://www.who.int/tb/laboratory/policy_statements /en/)
[7] Lawn SD, Nicol MP. Xpert® MTB/RIF assay: development, evaluation and implementation of a new rapid molecular diagnostic for tuberculosis and rifampicin resistance. Future Microbiol 2011;6(9):1067-82.

[8] Central TB division (India). Revised National TB Control Programme. Technical and Operational Guidelines for Tuberculosis Control in India, 2016.

[9] Helb D, Jones M, Story E, et al. Rapid detection of Mycobacterium tuberculosis and rifampin resistance by use of on-demand, near-patient technology. Journal of Clinical Microbiology 2010;48(1):229-37.

[10] Annual Status Report. Central TB Division. Official website of the Revised National TB Control Programme, Directorate General of Health Services, Ministry of Health \& Family Welfare Government of India. 2015. http://www.tbcindia.org.

[11] Central TB Division (India). Revised National Tuberculosis Control Programme. Training Course for Program Manager (Modules1-4). April, 2011.

[12] Dewan R, Anuradha S, Khanna A, et al. Role of cartridge-based nucleic acid amplification test (CBNAAT) for early diagnosis of pulmonary tuberculosis in HIV. JIACM 2015;16(2):114-7.

[13] Sen T, Joshi SR, Udwadia ZF. Tuberculosis and diabetes mellitus: merging epidemics.J Assoc Physicians India 2009;57:399-404.

[14] Central TB Division. Official website of the Revised National TB Control Programme, Directorate General of Health Services, Ministry of Health \& Family Welfare Government of India. 2012. http://www.tbcindia.org.

[15] Theron G, Peter J, van Zyl-Smit RV, et al. Evaluation of the Xpert MTB/RIF Assay for the diagnosis of pulmonary tuberculosis in a high HIV prevalence setting. Am J Respir Crit Care Med 2011;184(1):13240.

[16] Geleta DA, Megerssa YC, Gudeta AN, et al. Xpert MTB/RIF assay for diagnosis of pulmonary tuberculosis in sputum specimens in remote health care facility. BMC Microbiology 2015;15:220.

[17] Agrawal M, Bajaj A, Bhatia V, et al. Comparative study of gene Xpert with ZN stain and culture in samples of suspected pulmonary tuberculosis. Journal of Clinical and Diagnostic Research 2016;10(5):DC09-DC12.

[18] Sharma SK, Kohli M, Yadav RN, et al. Evaluating the diagnostic accuracy of Xpert MTB/RIF assay in pulmonary tuberculosis. PLoS One 2015;10(10): e0141011.

[19] Sowjanya DS, Behera G, Reddy VVR, et al. CBNAAT: a novel diagnostic tool for rapid and specific detection of Mycobacterium tuberculosis in pulmonary samples. International Journal of Health Research in Modern Integrated Medical Sciences 2014;1:28-31.

[20] Boehme CC, Nabeta P, Hillemann D, et al. Rapid molecular detection of tuberculosis and rifampin resistance. N Engl J Med 2010;363(11):1005-15. 
[21] Van Rie A, Page-Shipp L, Scott L, et al. Xpert(®) MTB/RIF for point-of care diagnosis of TB in high-HIV burden, resource-limited countries: hype or hope? Expert Rev Mol Diagn 2010;10(7):937-46.

[22] Carriquiry G, Otero L, Gonza'lez-Lagos E, et al. A diagnostic accuracy study of Xpert(®)MTB/RIF in HIV-positive patients with high clinical suspicion of pulmonary tuberculosis in Lima, Peru. PLoS One 2012;7(9):e44626.
[23] Vadwai V, Boehme C, Nabeta P, et al. Need to confirm isoniazid susceptible cases in Xpert MTB/RIF rifampicin susceptible cases. Indian J Med Res 2012;135(4):560-1. 